\title{
Iconic Retrieval by Contents from Databases of Cardiological Sequences
}

\author{
Gianfranco Congiu ${ }^{\dagger}$, Alberto Del Bimbo ${ }^{\dagger \ddagger}$, Enrico Vicario ${ }^{\dagger}$ \\ † Dipartimento Sistemi e Informatica, Universitá di Firenze \\ ¥ Dipartimento Elettronica per l'Automazione, Universitá di Brescia \\ email: congio,vicario@aguirre.ing.unifi.it, alberto@idea.ing.unibs.it
}

\begin{abstract}
The inherent complexity of retrieval by contents of image sequences imposes the use of appropriate techniques supporting the representation of the contents and the specification of content-oriented queries. A number of such techniques have been proposed in the literature but all of these consider images representing un-deformable rigid shapes. In this paper, the case of time-varying imaged curves is addressed with reference to a database of cineangiographic reports. Qualitative static and dynamic shape descriptors are introduced to provide a symbolic representation of the evolution of deformable shapes, and a language based on Interval Logic is presented which supports the expression of content-oriented queries. A prototype system is described which exploits these indexing and querying languages to support retrieval by contents from a database of cineangiographic reports through visual iconic interaction.
\end{abstract}

\section{Keywords}

Image Sequence Retrieval by Contents, Image Sequence Indexing, Deformable Imaged Shapes, TimeVarying Curves, Visual Iconic Querying.

\section{INTRODUCTION}

With the emergence of multimedia applications, a growing interest is being focused on databases of pictorial data such as images, image sequences and video. In these databases, the lack of a native structured organization and the inherent visuality of the information contents advises against the use of conventional indexing and retrieval based on textual keywords. A more effective retrieval is achieved by directly referring to the visual contents of pictorial data.

Iconic indexes, which stand for imaged objects, object features, and relationships, have been proposed in (Tanimoto 1976) to support image retrieval by contents. The use of iconic indexes naturally supports visual querying through iconic interaction as it permits the 
iconic expression of the distinctive features of data to be retrieved. This querying approach improves the user's involvement in the querying activity and reduces human/computer distance in the access to the database.

A number of techniques have appeared in the literature which deal with iconic indexing and visual querying of single images, taking into account different facets of the informative contents of pictorial data, such as picture color distribution (Swain 1991), object texture organization (Niblack 1993), similitude with visual sketches (Niblack 1993),(Hirata 1992),(Del Bimbo 1994), and semantic relationships between imaged objects (Chang 1984), (Chang 1987), (Chang-JVLC),(Roussoupoulos 1988), (Del Bimbo 1993).

As opposite to the large number of experiences on single images, only a few techniques have been proposed for indexing and querying of image sequences and video. Different proposals consider the sequencing of color histograms (Nagasaka 1992), handmade annotations reflecting the occurrence of significant situations (Davis 1993), semantic networks capturing high-level spatio-temporal interactions (Walter 1992), symbolic projections capturing the temporal evolution of spatial relationships among imaged objects (Arndt 1989),(Del Bimbo 1996). Moreover, all of these attempts deal with image sequences representing rigid objects and they cannot be extended to the case of image sequences where motion includes the deformation of a time-varying shape. This is the case of image sequences of fluids, gases, or deformable solid objects, and it finds a major relevance in the context of biomedical applications.

In this paper we present an original technique for symbolic indexing and querying of the contents of image sequences representing time-varying curves. This technique relies on a formal nucleus made up of two languages which support the concrete description of the actual contents of data at their storage time, and the specification of abstract data properties expressing content-oriented queries. The two languages are related by the fact that they permit the automatic checking of abstract queries against concrete data descriptions.

The definition of the two languages used to describe data and to express queries is largely conditioned by the type of data managed in the retrieval system and by the type of user interface that this is supposed to offer. To give concreteness to the treatment, the specific case of curves deriving from medical cardiologic reports will be addressed. Qualitative static and dynamic shape descriptors are introduced to provide a symbolic representation of these type of curves, and a language is presented which supports the expression of queries on their contents. A prototype system is described which exploits these indexing and querying languages to support retrieval by contents from a database of cineangiographic reports. The user interface of this system relies on a visual language allowing physicians to compose queries on geometric and temporal properties of reports to be retrieved through iconic interaction. 


\section{CONCRETE DATA DESCRIPTION}

A cineangiographic report consists of a sequence of images sampling the evolution of a section of the left ventricular heart wall along a complete beating period. For each individual sample, conventional edge detection and segmentation techniques partially support the automatic extraction of the contour of the ventricular wall. This extraction is usually completed through the manual marking of the contour, and through the automatic computation of its numerical representation in the form of a planar curve mapping a finite number of points of a conventional parametric axis onto a set of points of the Cartesian plan (see Fig.1).

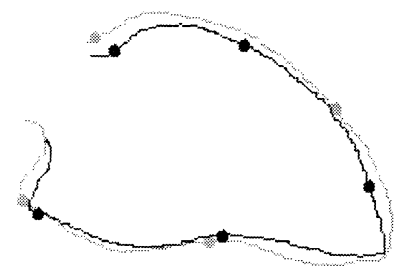

Figure 1 Two subsequent time samples in a cineangiographic diagnostic report. Conventional diagnostic equipments represent the complete beating period through 14-28 time samples.

The numerical representation of cineangiographic reports allows for digital storage and visual display, but not for a direct access in contents-oriented retrieval operations. To overcome this problem, cineangiographic reports can be associated with symbolic representations extracting the relevant features of sample contents while leaving undefined unnecessary numerical details.

Matching the native organization of cineangiographic reports, one such representation can be accomplished through a separate description of the static contents of individual samples and of the tracking information capturing the sequence evolution.

\subsection{Static Curve Description}

Studies on human perceptual processing show that the observation of curves does not focus on the position of individual points but rather on their organization within continuous regions characterized by homogeneous features (Haber 1983)(Palmer 1983). This yields a two-layered knowledge model in which the overall shape of a curve is segmented in subcurves reproducing a small number of basic elementary patterns (Leyton 1982). Different 
sets of such basic patterns and different partitioning criteria have been proposed in the literature (Leyton 1982)(Marshall 1989).

In the codon approach (Richards 1984), a curve is regarded as a chain of codons:

curve $:=$ codon $\mid$ curve $_{1} \rightarrow$ codon

each codon being comprised of the curve points between two subsequent minima of the bending function. The codon approach appears to be well suited for the case of cineangiographic reports as its orientation towards the characteristics of the bending function naturally matches the relation between the shape of the heart contour and the partitionment of the heart body into physiologic regions characterized by different values of internal muscular tension forces. Codon-based descriptions are also independent of either the orientation or the zooming factor that are employed during the imaging process.

$[0+]$
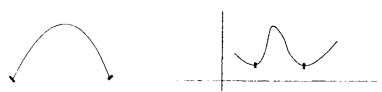

$[0-]$
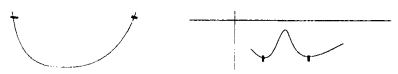

$[1+]$
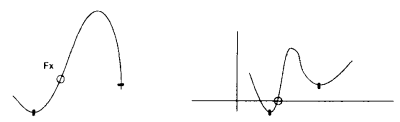

$[1-]$
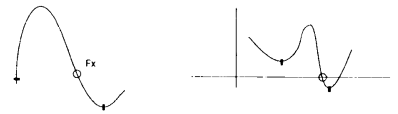

[2-]
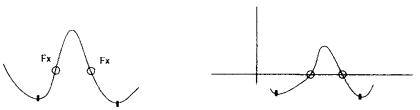

Figure 2 A codon is a sub-curve delimited by two subsequent minima of the bending function. Codons can be classified on the basis of the sign of the bending function in the extrema and of the number of flex points between them. There exists five different combinations: two with no flex points, two with one flex, and one with two flex points. 
Codons can be associated with static attributes providing a symbolic characterization of their geometric shape. In the case of cineangiographic reports, each codon is associated with a type reflecting the pattern of the bending function, and with three shape attributes of symmetry, bending, and extension:

static attributes $:=$ type + bending + symmetry + extension

The codon type reflects the five possible patterns of the sign of the bending function along the codon (see Fig.2). Symmetry accounts for the position of the bending maximum with respect to the middle point of the codon; bending is a qualitative representation of the maximum value of the bending function with respect to symbolic thresholds; and extension captures the linear distance between the extrema of the codon.
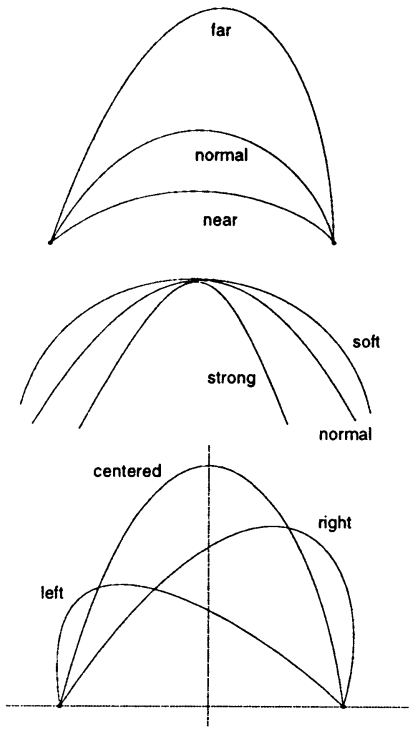

Figure 3 The three characterizing attributes of symmetry, bending, and extension, and their corresponding symbolic values.

\subsection{Dynamic Curve Description}

The static attributes of codons provide a description of geometric static features of the individual samples of a cineangiographic curve. In order to capture the temporal evolution of the overall report, codons belonging to subsequent samples are associated through a parent-child tracking relation (see Fig.4). The tracking relation is usually one-to-one (i.e. 
each codon of sample $j$ is biunivocally associated with a single codon of sample $n+1$ ). However, due to the appearance or disappearance of bending minima, cases of splitting (i.e. a codon of sample $j$ is associated with multiple codons of sample $j+1$ ) or merging (i.e. multiple codons of sample $j$ are associated with a single codon of sample $j+1$ ) are also possible.

To cope with merging and splitting, the tracking relation is more easily derived from the parent/child relation among codons extrema (i.e. minima of the bending function): if $\left\{m_{k}^{j}\right\}_{k=1}^{K_{j}}$ denotes the sequence of bending minima in the $j^{\text {th }}$ sample, a tracking relation for a sequence of $J$ samples is a sequence of $J$ relations $\gamma_{j}$ associating minima through subsequent samples:

$\gamma_{j}:\left\{m_{k}^{j}\right\}_{k=1}^{K_{j}} \rightarrow\left\{m_{k}^{j-1}\right\}_{k=1}^{K_{j}-1} \bigcup\{n e w\}$

Specifically, $\gamma_{j}\left(m_{k}^{j}\right)=m_{h}^{j-1}$ means that the minimum $m_{k}^{j}$ in sample $j$ is the evolution of minimum $m_{h}^{j-1}$ in sample $j-1$, and $\gamma_{j}\left(m_{k}^{j}\right)=$ new means that the minimum $m_{k}^{j}$ is newly appeared in sample $j$. All the minima of sample $j-1$ that are not associated with any minimum of sample $j$ are assumed to be disappeared.

Depending on the aliasing yielded by the time sampling, the association of bending minima through subsequent frames is carried out through an heuristic decision which basically consists in minimizing a cost function accounting for the curvilinear abscissa displacement between related minima (Congiu 1994). Specifically, if $\operatorname{dist}\left(m_{k}, m_{h}\right)$ is a distance between minima belonging to subsequent frames, the tracking relations $\gamma_{j}$ are chosen so as to minimize the sum of distances between related minima:

$\operatorname{cost}=\sum_{j=1}^{J} \sum_{k=1}^{K_{j}} \operatorname{dist}\left(m_{k}^{j}, \gamma_{j}\left(m_{k}^{j}\right)\right)$

Once the tracking relation has been reconstructed, the static characterization of codons (see Sect.2.1) can be refined through the introduction of two dynamic attributes derived from the analysis of relationships among related codons in subsequent frames:

dynamic attributes $:=$ straining + region

The straining attribute captures the instantaneous expansive/contractive trend of each codon, and it is derived by comparing the position of the codon with that of its parents in the tracking relation (see Fig.5). It is worth remarking that the straining attribute provides a local representation of the evolution of the curve in that it refers to the individual codons. In general, different straining trends may be exhibited by different codons of the same sample. In the cineangiographic diagnostic activity, the simultaneous presence of strongly 


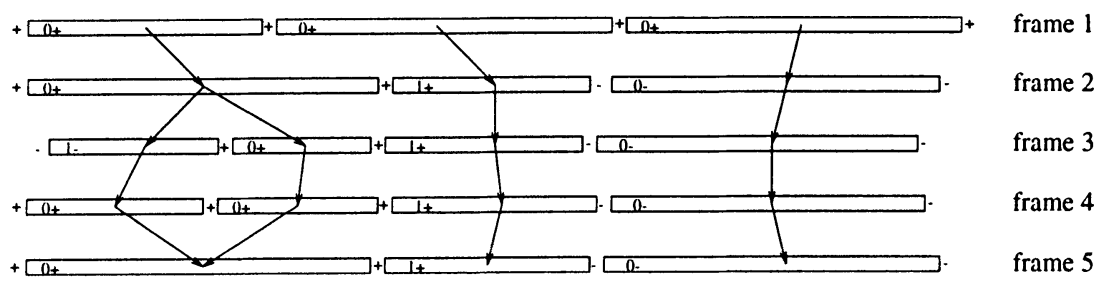

Figure 4 Codon tracking for six samples of a cineangiographic report. For each sample, the curve is represented in curvilinear abscissa and it is segmented into codons that are tracked along subsequent samples. The first codon of the first sample is tracked onto the first codon of the second sample. In turn, this is split into the first and second codon of the third sample. The first and second codon of the fourth sample are merged into the first codon of the fifth sample.

differentiated straining trends is a condition of main relevance as it points out important pathologies.

The region attribute is strictly related to a specific properties of the heart wall contour which is natively partitioned in three different anatomic regions, usually referred to as anterior, apical and inferior. Reflecting the physiologic separation between the three parts, the tracking relation identifies three disjoint sets of codons which are not connected through any merging or splitting event. This permits the automatic attribution of each part of each sample of the curve to one of the three anatomic regions. Please note that each codon of each sample is assigned to one of the three regions on the basis of a global analysis of the entire heart-beating period. For this reason, the belonging of a codon to a certain anatomical region is regarded as a dynamic attribute.

\section{ABSTRACT QUERY SPECIFICATION}

In retrieval by content systems, queries are expressed through an abstract characterization of the expected distinctive features of the concrete description of data in the database. In the case of time-varying curves, this characterization involves both the geometric features of the curve at individual time instants and the ordering relationships between the time intervals in which these features are maintained.

According to the organization of concrete descriptions of individual static samples (cf. 

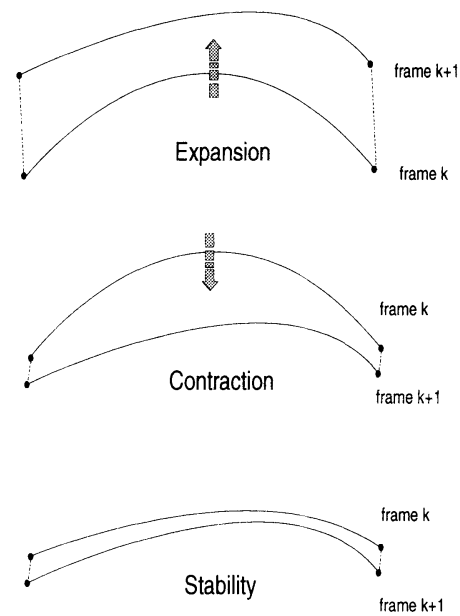

Figure 5 The straining attribute captures the instantaneous straining trend of an individual codon. It is assumed to be expansive, stable or contractive depending on the relation between the areas subtended by the codon and its successors. Each of these three conditions is further specialized by an intensity qualifier which may take the values soft, normal or strong.

Sect.2.1), geometric features can be expressed as a Boolean combination of basic propositions stating the existence of a codon with a certain set of attributes:

$$
\begin{aligned}
& \theta:=\text { basic }\left|\neg \theta_{1}\right| \theta_{1} \wedge \theta_{2} \\
& \text { basic }:=\text { "there exists a codon with attributes } p "
\end{aligned}
$$

where $p$ is any possible evaluation for the static and dynamic attributes of a codon.

The temporal evolution of the curve can now be described in terms of the ordering relationships between the lasting intervals in which geometric features of interest are maintained (see Fig.6). To this end, if $I(\theta)$ stands for the any interval in which the property $\theta$ is maintained, any such ordering relationship can be expressed in the form

$\Theta:=I\left(\theta_{1}\right) \sim I\left(\theta_{2}\right)\left|\neg \Theta_{1}\right| \Theta_{1} \wedge \Theta_{2}$

$\sim$ standing for any of the 13 possible ordering relationships which may occur between a 
couple of intervals (Allen 1983). These relationships, which are pictured in Fig.7, distinguish all the possible conditions of precedence and coincidence between any two extremes of the two intervals.
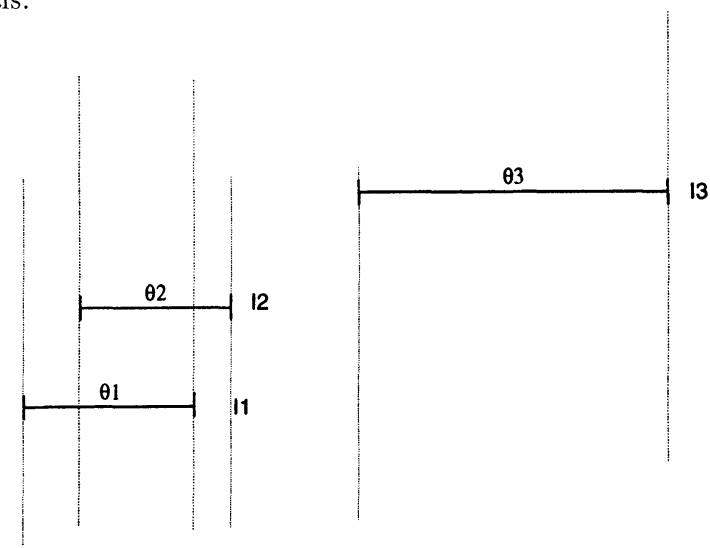

Figure 6 A temporal evolution characterized by three conditions $\theta_{1} \theta_{2}$ and $\theta_{3}$. Condition $\theta_{1}$ begins first and lasts until overlapping the first part of $\theta_{2}$. Condition $\theta_{3}$ occurs after the end of $\theta_{2}$. The overall timing is captured by the sentence $I\left(\theta_{2}\right) \sim_{3} I\left(\theta_{1}\right) \wedge I\left(\theta_{3}\right) \sim_{1} I\left(\theta_{2}\right)$.

\section{A SYSTEM FOR VISUAL SEARCH AND RETRIEVAL}

The two languages described in Sects. 2 and 3 have been exploited as formal nucleus of a system for retrieval by contents of cineangiographic reports supporting medical personnel in the diagnostic analysis of cineangiographic reports *

At storage time, a cineangiographic report is given as a sequence of raw digital images. These are reduced in numerical representation through a semi-automatic process, which includes automatic edge detection and segmentation, manual marking of the leftventricular contour, and automatic derivation of the chain code representation and of the bending function of contours of individual samples (Baroni 1992). The numerical representation is stored in the system along with a symbolic content representation expressed according to the description format expounded in Sect.2.

In the retrieval stage, the user expresses content-oriented queries through a visual iconic interface. Specifically, the user defines the distinctive conditions which characterize se-

\footnotetext{
*The system presently runs on an IBM R6000 workstation.
} 


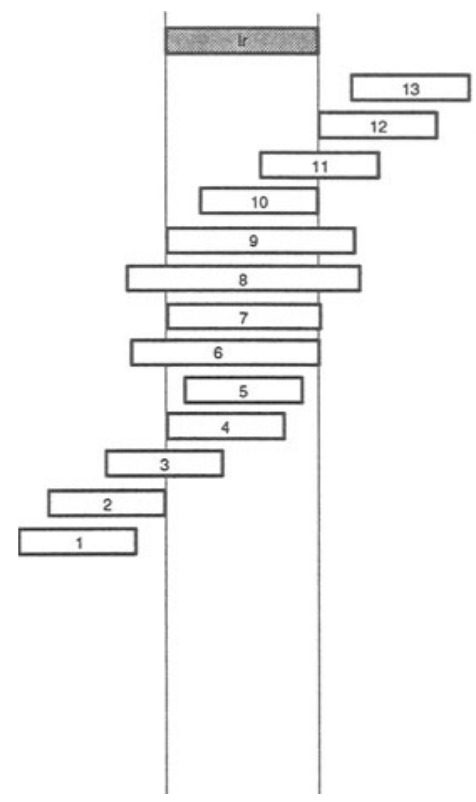

Figure 7 The 13 possible mutual positions of two intervals. Disjunctive composition of the individual positions permits the expression of relevant ordering conditions such as after (\#13), before (\#1), during $(\# 4 \vee \# 5 \vee \# 10)$ and overlapping $(\# 3 \vee \# 11)$

quences to be retrieved and defines the ordering relationships among the lasting intervals of each of these conditions.

Visual queries are automatically interpreted and translated into formulae of the querying language described in Sect.3. These formulae are then checked against the symbolic description of the reports stored in the database, and, finally, matching sequences are displayed on the screen.

\subsection{Visual Querying}

The basic graphic objects that are employed in the visual expression of queries are icons and lines. Following an approach similar to that used in (Kutty 1993), these objects are arranged on the screen to express visual queries that will be automatically interpreted by the system and translated into formulae of the querying language described in Sect.3.

Icons represent significant conditions in the evolution of a report, such as an expansive straining or the presence of a sharp bending in some of the codons of the cineangiographic curve. Multiple icons can be grouped together to express the Boolean composition of 
multiple events: conjunctive and disjunctive compositions are expressed by arranging icons in rows and columns, respectively, while icon crossing expresses negation (see Fig.8).

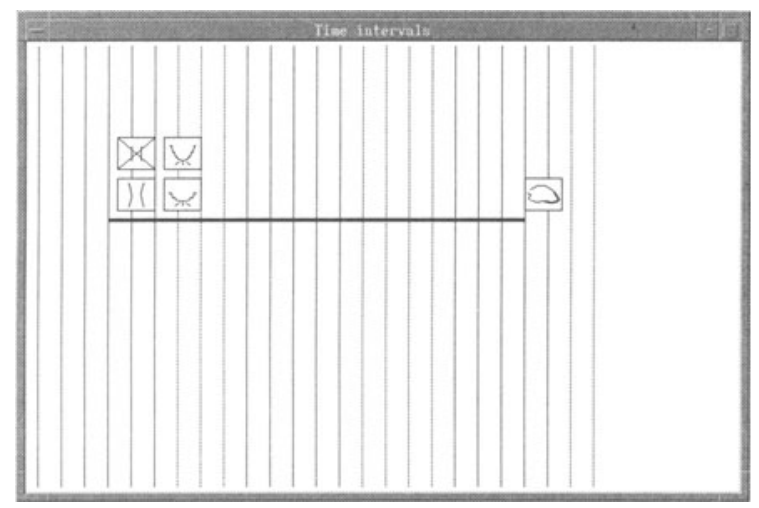

Figure 8 The horizontal line stands for the time interval in which the condition expressed by the icon arrangement on the left hand side is maintained in the region specified by the icon on the right hand side. Formally, it denotes the interval $I(\theta)$, where theta is expressed as "there exists a codon with attributes $p^{\prime \prime}$, with $p=($ region $=$ apical $) \wedge(($ straining $=$ contractive $\wedge$ bending $=$ soft $) \vee(\neg($ straining $=$ stasis $) \wedge$ bending $=$ medium $)$ ).

Lines are used to denote the lasting time intervals in which events and event compositions occur. To this end, lines are drawn on the screen and annotated with the iconic representation of the event composition. Each line is also annotated with a special icon denoting the anatomic region in which the event composition occurs. As a particular case, lines can be annotated with any of two icons representing the two reference phases of systolic contraction and diastolic expansion of the heart beating cycle (see Fig.9).

The mutual positioning of event lines on the screen defines the temporal ordering among the lasting intervals of distinctive events. To permit the expression of don't care conditions in the interpretation of this mutual positioning, each line is associated with one or more colors, and the mutual position between intervals which do not share any common color is ignored in the interpretation of the visual arrangement.

Once lines and icons have been placed on the screen, the system interprets their visual arrangement and translates it into a formula of the querying language described in Sect.3. This occurs through two distinct phases. First, the mutual positioning of event intervals sharing one or more grouping colors are analyzed and classified into one of the 13 possible mutual ordering relations of Fig.7. Afterwards, icon compositions associated with 


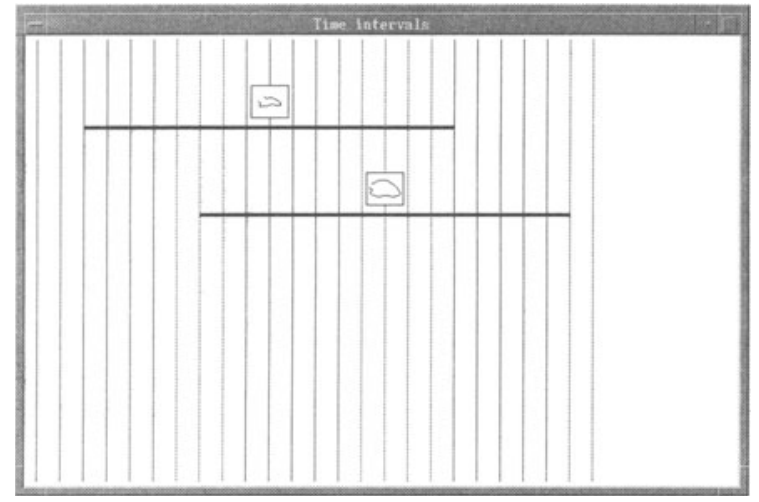

Figure 9 Two lines representing the lasting intervals of the systolic (upper line) and diastolic (lower line) physiologic phases of the heart beating period.

each interval are parsed and translated into interval formulae expressed according to the language of Sect.3.

The model-checking algorithm and the parser work together interactively. For each reduced grammar level corresponding actions are produced for matching with possible intervals coming from the symbolic representations related to image sequence stored in the database, and standing as candidates.

After decoding the textual sentence, the intervals which meet the temporal relationships recognized by the first visual parser are selected. These formulae are then checked against the concrete descriptions of the sequences in the database, and matching reports are retrieved and displayed on the screen.

\subsection{An Example}

For the sake of concreteness, let us consider an operation example in which the system is used to retrieve reports characterized by an outward motion in the apical region during the systolic phase. This is a pathologic behavior in that, during the systolic phase, the entire wall contour should exhibit a contractive motion.

Fig. 10 reports the visual query capturing the phenomenon of interest. The upper line is annotated with the systolic icon thus denoting the time interval in which the heart should expand. The lower line is annotated with two icons representing an expansive strain (in the left hand side of the line) and the apical region (in the right hand side of the line) 
so as to represent a time interval in which part of the apical region exhibits an expansive motion. The two lines are associated with a common color so as to make significant the mutual positioning of the two intervals.

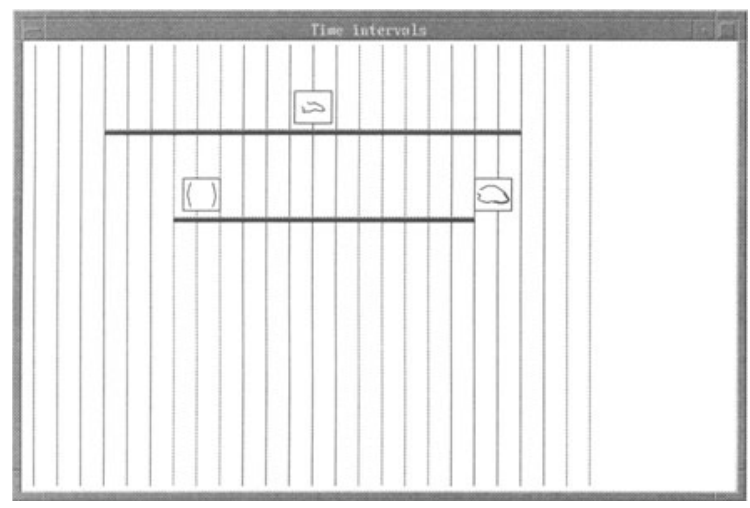

Figure 10 A visual query expressing a contractive strain in the apical region during the systolic phase: $I\left(\theta_{1}\right) \sim_{5} I\left(\theta_{2}\right)$ with $\theta_{1}=$ systolic phase and $\theta_{2}=$ "there exists a codon with attributes $p$ with $p=($ region $=$ apical $) \wedge($ strain $=$ expansive $)$.

The system interpretation of the overall arrangement produces a query statement which causes the retrieval of the reports characterized by a time interval which is contained in the systolic phase and which exhibits a contractive motion in the apical region. When retrieval is completed, reference names of retrieved reports are listed on a menu which permits selection and display on the screen of reports of specific interest for the user (Fig.11).

A more selective retrieval is obtained if the query is refined by the addition of further conditions. In particular, Fig.12 shows the query which is obtained adding the request for an inward motion in the apical region during the diastolic phase. Two further lines are added (in the bottom part of the screen), one standing for the diastolic phase and the other for the lasting interval of the contractive motion in the apical region. These two lines are associated with a common color different from that associating the previous two lines. As a result, the system interpretation will take care of the ordering between the two new lines and between the two old lines, but it will not consider the ordering between new and old lines. Again, after the interpretation of the query, retrieval is carried out and matching reports are listed and displayed on the screen. Of course, retrieved sequences are a subset of the ones found by the previous query (Fig.13). 

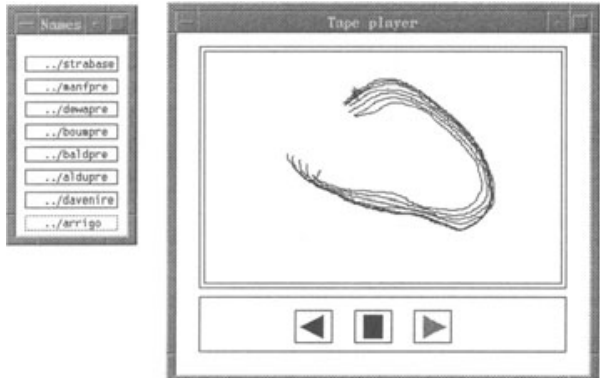

Figure 11 The list of retrieved reports (left hand side menu) permits selection and display (right hand side window) frame by frame of reports of interest.

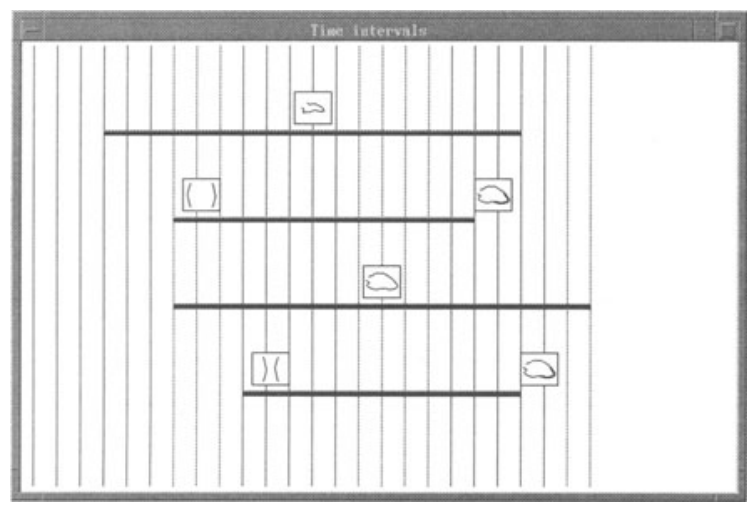

Figure 12 A refinement of the query of Fig.10. In this case, the query expresses the existence an expansive strain in the apical region during the systolic phase and a contractive strain, again in the apical region, during the diastolic phase. The textual expression of the statement is: $\left(I\left(\theta_{1}\right) \sim_{5} I\left(\theta_{2}\right)\right) \wedge$ $\left(I\left(\theta_{3}\right) \sim_{5} I\left(\theta_{4}\right)\right)$, where $\theta_{1}$ and $\theta_{2}$ are defined as in Fig.10, while $\theta_{3}=$ diastolic phase and $\theta_{4}=$ "there exists a codon with attributes $p$ with $p=($ region $=$ apical $) \wedge($ strain $=$ contractive $)$. 


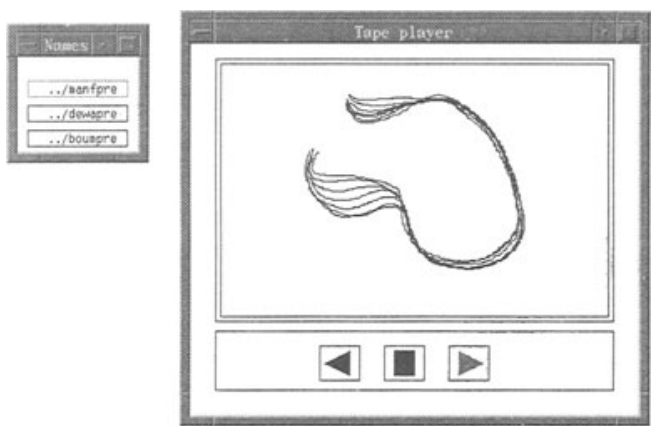

Figure 13 Retrieval result for the example query of Fig.12.

\section{CONCLUSIONS}

Two original languages for the concrete description and the abstract specification of the contents of images representing time-varying curves have been presented. The concrete description language supports the symbolic representation of the spatial and temporal evolution of a curve, while the abstract specification language permits the expression of properties characterizing a possible multiplicity of curves. The two languages are related by the fact that they permit abstract specifications to be automatically checked against concrete descriptions.

This automatic checking has been exploited in a retrieval system supporting visual iconic querying by contents from databases of biomedical image sequences representing cineangiographic reports. In this system, the user is allowed to compose visual queries describing distinctive features of visual data to be retrieved through the use of a two-level iconic interface. At the first level, the user arranges iconic indexes to express static geometric features occurring in the individual images of reports to be retrieved. At the second level, the temporal ordering with which static features occur is expressed by associating static indexes with time lines representing their lasting intervals.

While the treatment of this paper has been focused on the specific case of medical reports handled by the system, the use of two dual languages for the concrete description of stored data and the abstract specification of content queries can be extended to a large variety of applications concerned with retrieval by contents of image sequences containing non-rigid, time-varying curves. 


\section{REFERENCES}

Allen,J.F. (1983) "Maintaining Knowledge about Temporal Intervals," Comunications of the ACM, Vol.26, n.11, Nov. 1983.

Arndt, T, Chang,S.K. (1989) "Image Sequence Compression by Iconic Indexing", IEEE VL '89 Workshop on Visual Languages, Roma, Italy, Sept.1989.

Baroni,M., Barletta,G. (1992) "Digital Curvature Estimation for Left Ventricular Shape Analysis," Image and Vision Computing, 1992.

Binaghi,E. (1992) "Indexing and Fuzzy Logic-Based Retrieval of Color Images," in IFIP Transactions A-7, Visual Database Systems II, Knuth, Wegner (Eds.), Elsevier Pub. 1992.

Chang,S.K. Liu,S.H. (1984) "Picture indexing and Abstraction Techniques for Pictorial Databases", IEEE Transactions on Pattern Analysis and Machine Intelligence, Vol.6, No.4, July 1984.

C'hang,S.K., Shi,Q.Y., Yan,C.W. (1987) "Iconic Indexing by 2-D Strings", IEEE Transactions on Pattern Analysis and Machine Intelligence, Vol.9, No.3, July 1987.

Chang,S.K., Hou, T.Y. Hsu,A. (1992) "Smart Image Design for Large Image Databases", Journal of Visual Languages and Computing, Vol.3, No.4, Dec.1992

Congiu,G. (1994) "Rappresentazione e Ricerca Visuale di Sequenze di Immagini Cardiologiche (In Italian)," Doctoral Thesis, A.Del Bimbo (Adv.) Dept.Sistemi e Informatica, Univ. Firenze, Italy, July, 1994.

Davis,A. (1993) "Media Streams, an Iconic Visual language for Video Annotation," Telektronik, No.4, 1993, (also appeared in reduced version in Proc.IEEE VL'93 Workshop on Visual Languages, Bergen, Norway, Aug.1993).

Del Bimbo,A., Vicario,E., Zingoni,D. (1996) "Symbolic Representation and Visual Querying of Image Sequences Usinral Logic," accepted for publication in the IEEE Transactions on Knowledge and Data Engineering.

Del Bimbo,A., Campanai,M., Nesi,P. (1993) "A Three-Dimensional Iconic Environment for Image Database Querying," IEEE Transactions on Software Engineering, Vol.19, No.10, Oct.1993.

Del Bimbo,A., Pala,P., Santini,S. (1994) "Visual Image Retrieval by Elastic Deformation of Object Shapes," Proc. IEEE VL'94, Int.Symp. on Visual Languages, St.Louis, MI, Oct.1994.

Haber,R.N. (1983) "Stimulus Information and Processing Mechanism in Visual Perception," in Human and Machine Vision, Academic Press, New York, 1983.

Hirata,K. Kato,T. (1992) "Query by Visual Example: Content-Based Image Retrieval," In Advances in Database Technology - EDBT'92, A.Pirotte, C.Delobel, G.Gottlob (Eds.), Lecture Notes on Computer Science, Vol.580, Springer Verlag, Berlin, 1992.

Kutty,G., Dillon,L.K., Moser,L.E., Melliar-Smith,P.M., Ramakrisnha,Y.S. (1993) "Visual Tools for Temporal Reasoning," in Proc. IEEE VL'93, Int.Symp. on Visual Languages, 1993.

Lee,S. Shan,M.K., Yang,W.P. (1989) "Similarity Retrieval of Iconic Image Database," Pattern Recognition, Vol.22, No.6, 1989.

Leyton,M. (1982) "Shape and Casual History," in Visual Form, Plenum Press, 1982.

Marshall,S. (1989) "Review of Shape Coding Techniques," in Image and Vision Computing, Nov.1989.

Nagasaka,A., Tanaka,Y. (1992) "Automatic Video Indexing and Full Video Search for Object Appearances," in IFIP Transactions, Visual Database Systems II, Knuth, Wegner (Eds.), Elsevier Pub. 1992.

Niblack,W. et alii, (1993) "The QBIC Project: Querying Images by Content Using Color, Texture and Shape," Res.Report 9203, IBM Res.Div. Almaden Res.Center, Feb.1993.

Palmer,S.E. (1983) "The Psychology of Perceptual Organization,: a Transformational Approach," in Human and Machine Vision, Academic Press, New York, 1983. 
Richards,W., D.D.Hoffman,D.D. (1984) “Codon Constraints on Closed 2D Shapes," in Computer Vision II, Natural Computation Group, M.I.T., Cambridge, 1984.

Roussopoulos,N., Faloutsos,C., Sellis,T. (1988) "An Efficient Pictorial Database System for PSQL", IEEE Transactions on Software Engineering, Vol.14, No.5, May 1988.

Swain,M.J., Gallard,D.H, (1991) "Color Indexing," Int.Journal of Computer Vision, Vol.7, No.1, 1991.

Tanimoto,S.L. (1976) "An Iconic/Symbolic Data Structuring Scheme," in Pattern Recognition and Artificial Intelligence, C.H.Chen (Ed.), New York Academic, 1976.

Walter,I.M., Sturm,R., Lockemann,P.C. (1992) "A Semantic Network Based Deductive Database System for Image Sequence Evaluation," in IFIP Transactions, Visual Database Systems II, Knuth, Wegner (Eds.), Elsevier Pub. 1992.

\section{BIOGRAPHIES}

Gianfranco Congiu was born in Firenze, Italy, in 1967. He received the doctoral degree in Electronics Engineering from the Università di Firenze, Italy, in 1994. He is currently with the Department of Sistemi e Informatica of the Università di Firenze with a grant funded by Bassilichi S.P.A., Florence, Italy. His research activities are in the field of image representation, indexing retrieval with a specific interest in databases of dynamic images.

Alberto Del Bimbo was born in Firenze, Italy, in 1952. He received the doctoral degree in Electronics Engineering from the Università di Firenze, Italy, in 1977. He was with IBM ITALIA from 1978 to 1988. He is Full Professor of Computer Systems at the University of Brescia and the University of Florence, Italy. Dr. Del Bimbo is a member of IEEE and of the International Association for Pattern Recognition (IAPR). He is in the board of the IAPR Technical Committee n. 8 (Industrial Applications) and the Vice-President of the IAPR Italian Chapter. He presently serves as Associate Editor of Pattern Recognition Journal and of the Journal of Visual Languages and Computing. His research interests and activities are in the field of image analysis, image databases, visual languages and virtual reality.

Enrico Vicario was born in Firenze, Italy, in 1965. He received the doctoral degree in Electronics Engineering and the Ph.D. in Computer Science from the Università di Firenze, Italy, in 1990 and 1994. He is currently Researcher with the Department of Sistemi e Informatica of the Università di Firenze. His research activities are in the field of software engineering, with a particular interest in visual formalisms, specification languages and validation techniques for time-dependent systems. 